Supplement of Geosci. Model Dev., 11, 3261-3278, 2018

https://doi.org/10.5194/gmd-11-3261-2018-supplement

(C) Author(s) 2018. This work is distributed under

the Creative Commons Attribution 3.0 License.

(c) (1)

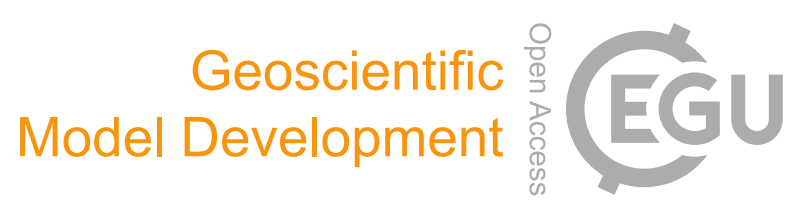

Supplement of

\title{
A parameterisation for the co-condensation of semi-volatile organics into multiple aerosol particle modes
}

M. Crooks et al.

Correspondence to: Matthew Crooks (matthewcrooks@ntlworld.com)

The copyright of individual parts of the supplement might differ from the CC BY 3.0 License. 
In this supplement, we discuss the necessity to initiate the DCP at a shorter time than it takes to get from an initial $90 \%$ RH up to cloud base. Figure 1 shows the variation in equilibrium condensed mass of SVOCs with relative humidity. Equilibrium absorptive partitioning theory (Barley et al. 2009) was used to calculate the condensed mass of SVOCs at different relative humidities when

5 seeded with a non-volatile mode of ammonium sulphate. The grey line shows the condensed mass at $90 \% \mathrm{RH}$ and there is little increase in condensed mass when the RH is increased to $95 \%$ shown by the dashed grey line. By $98 \%$ RH there is a more pronounced increase in total condensed mass (black dotted line) but it is not until $99 \%$ and $100 \%$ (dashed and solid black, respectively) that the majority of the highest volatility bin enters the condensed phase. It is the volatility bin with the highest $C^{*}$ value that contributes the most significantly to the additional condensed mass at cloud base. Figure 1 also suggests that there would little difference in performance of the DCP parameterisation if coupled to the cloud droplet activation scheme initiated from $90 \%$ or $95 \% \mathrm{RH}$ resulting from the initial condensed masses, $y_{i j}^{0}$, that are used. Some difference may be observed if the cloud droplet activation scheme were initiated from $98 \% \mathrm{RH}$ or above as there is an increase in equilibrium condensed mass at this relative humidity.

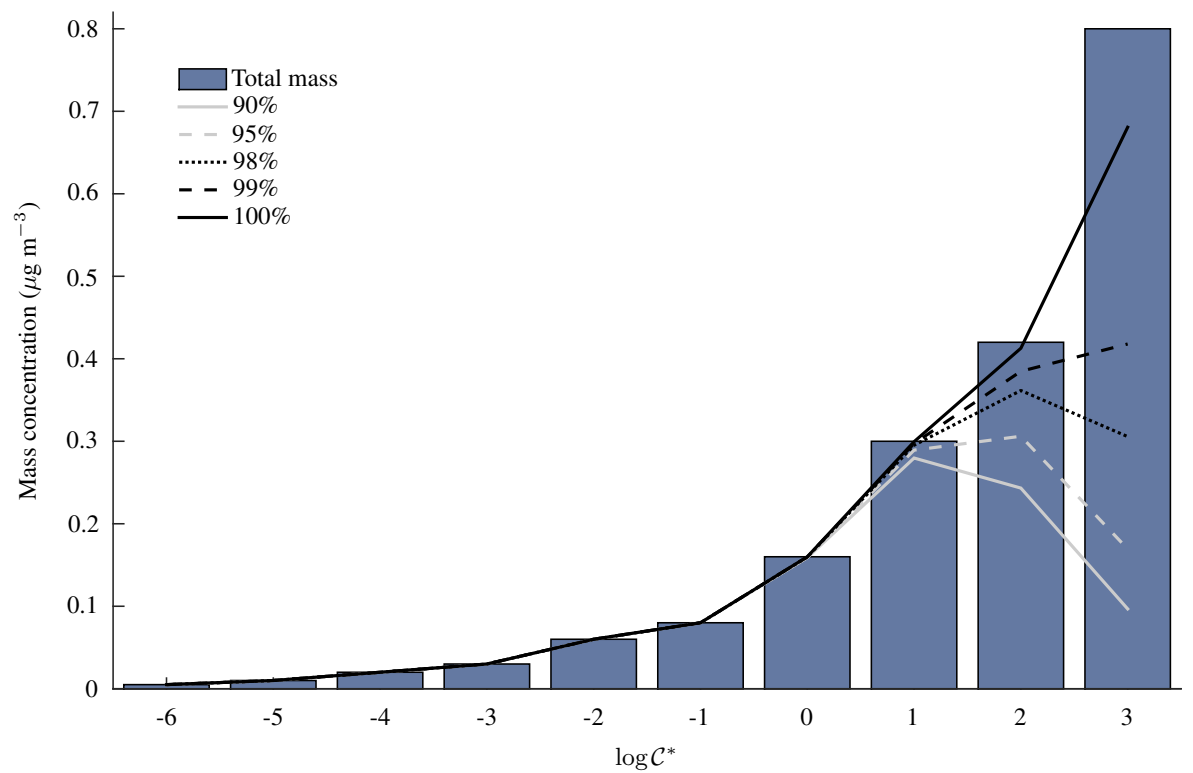

Figure 1. Variation in equilibrium condensed mass of SVOCs with relative humidity. A non-volatile mode of ammonium sulphate with lognormally distributed particle sizes was used as a seed aerosol with a number concentration of $500 \mathrm{~cm}^{-3}$, median diameter of $100 \mathrm{~nm}$ and geometric standard deviation of 0.5 . The bars show the total concentration of SVOCs in each volatility bin and the lines correspond to the total condensed mass calculated using equilibrium absorptive partitioning theory at the relative humidity values indicated in the legend.

We have, additionally, run the parameterisation with different cloud base times, $t_{c b}^{*}$, in the DCP for two of the different environmental scenarios described in Section 5 of the main paper. In all cases, an initial condensed mass, $y_{i j}^{0}$, is calculated using equilibrium absorptive partitioning theory at $90 \% \mathrm{RH}$. The time taken for the parcel of air to reach $100 \% \mathrm{RH}$ from $90 \% \mathrm{RH}, t_{c b}$, is rescaled to reflect the fact that the dominant condensation of SVOCs only occurs when the relative humidity approaches $100 \%$. Figures 2 and 3 show the performance of the parameterisation under different rescalings of $t_{c b}$.

In the Natural case, shown in Figure 2, there is little difference in the performance of the DCP for different values of $t_{c b}^{*}$ for the $10 \%$ organic mass fraction case. When $t_{c b}^{*}=t_{c b}$ there is almost perfect agreement between the parameterisation and the parcel model, with all other cases calculating 
slightly fewer CCN. For higher concentrations of SVOCs, the case when $t_{c b}^{*}=t_{c b}$ has a tendency to overpredict the number of CCN compared to the parcel model, while setting $t_{c b}^{*}=0.1 t_{c b}$ underpredicts. All other values of $t_{c b}^{*}$ are comparable to one another and are in good agreement with the parcel model.

At low concentrations of SVOCs in the Urban environment (top left plot of Figure 3), the cloud droplet activation parameterisation is largely insensitive to the value of $t_{c b}^{*}$. For higher SVOC concentrations, however, the number of CCN is highly sensitive to $t_{c b}^{*}$. As in the Natural case, $t_{c b}^{*}=t_{c b}$ overpredicts the number of CCN compared to the parcel model, while setting $t_{c b}^{*}=0.1 t_{c b}$ underpredicts. There is, again, little difference between the smaller values of $t_{c b}^{*}$. There is marginally better agreement for the case when $t_{c b}^{*}=0.01 t_{c b}$ and as such this is the value that we suggest using.
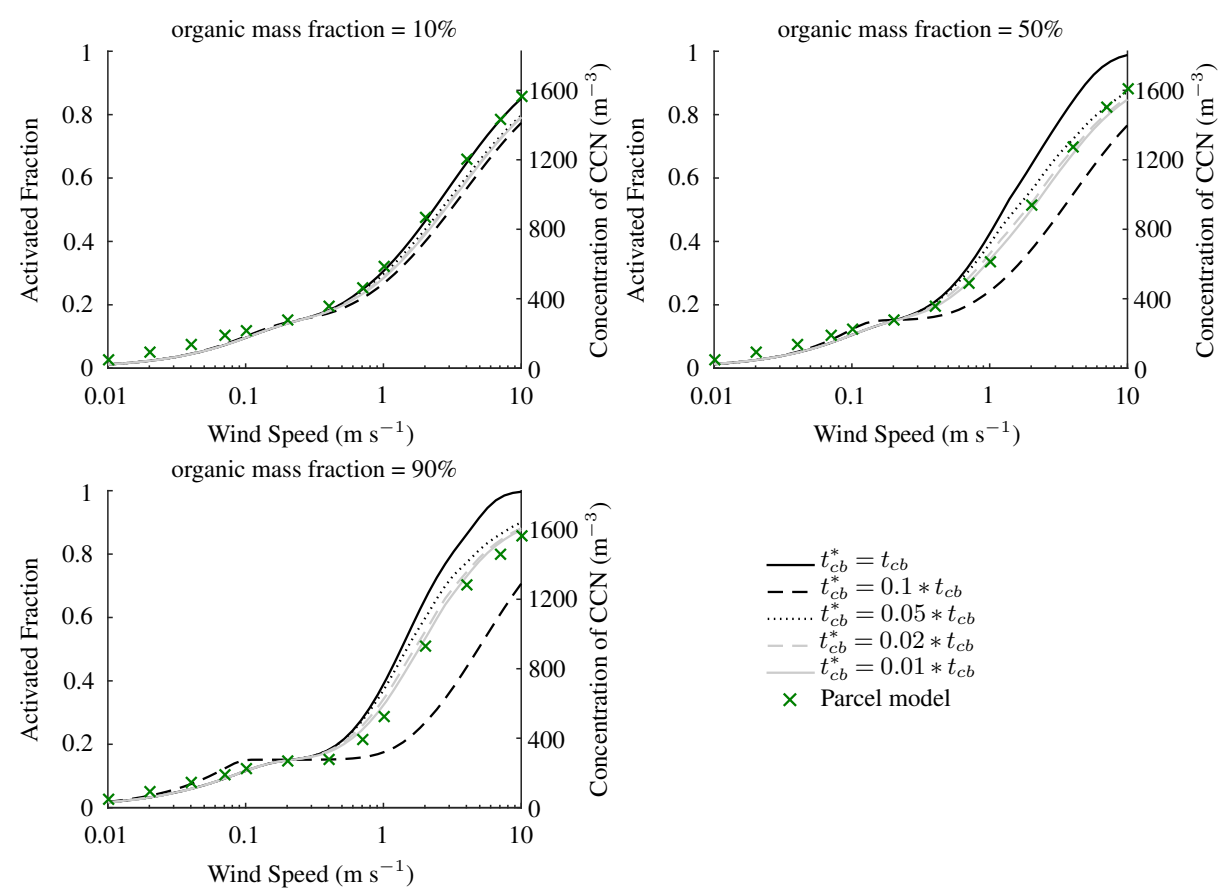

$$
\begin{aligned}
&-t_{c b}^{*}=t_{c b} \\
&--t_{c b}^{*}=0.1 * t_{c b} \\
& \ldots \ldots . . t_{c b}^{*}=0.05 * t_{c b} \\
&--t_{c b}^{*}=0.02 * t_{c b} \\
&-t_{c b}^{*}=0.01 * t_{c b} \\
& \times \quad \text { Parcel model }
\end{aligned}
$$

Figure 2. Performance of the cloud droplet activation parameterisation compared to the parcel model when the DCP is evaluated at different cloud base times, $t_{c b}^{*}$, for the Natural environment conditions. The legend shows the rescaling of $t_{c b}$ that is used to obtain the time, $t_{c b}^{*}$, at which the DCP is evaluated. The concentration of SVOCs used in each case is rescaled so that the organic mass fraction of the aerosol at $90 \% \mathrm{RH}$ is equal to the value indicated above each plot. Actual SVOC concentrations are given in Appendix C of the paper.

The time taken to reach cloud base, $t_{c b}$, used in Figures 2 and 3 are calculated from an initial relative humidity of $90 \%$. If the cloud droplet activation scheme is initiated from a different initial RH then a different value of $t_{c b}^{*}$ will need to be used to reflect this. A more universal method of calculating $t_{c b}^{*}$ is to define it as the time taken for a parcel of air to ascend from $99.9 \%$ to $100 \% \mathrm{RH}$. This includes dependence of initial temperature, pressure and $\mathrm{RH}$ as well as vertical updraft. 

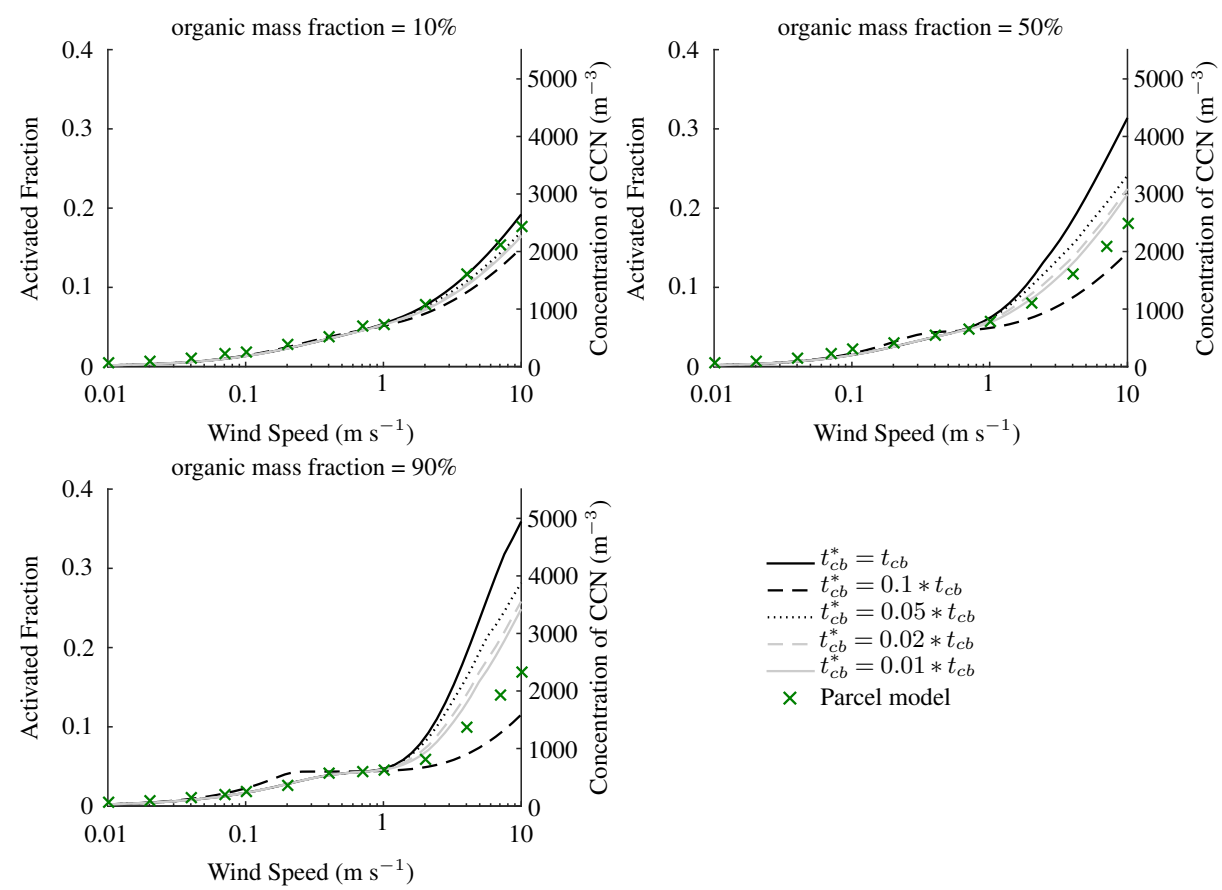

Figure 3. Same as Figure 2 but for the Urban environment conditions.

\section{References}

Barley, M., Topping, D. O., Jenkin, M. E., and McFiggans, G.: Sensitivities of the absorptive partitioning model of secondary organic aerosol formation to the inclusion of water, Atmospheric Chemistry and Phyics, 9, 2919-2932, 2009. 